\title{
45
}

\section{Evaluación Económica para la toma de decisiones sobre cobertura en salud: ¿qué debe saber el profesional de la salud?}

\author{
Manuel Antonio Espinoza \\ Departamento de Salud Pública, Unidad de Evaluación de Tecnologías en Salud \\ Escuela de Medicina, Pontificia Universidad Católica de Chile.
}

Resumen: Los sistemas de salud enfrentan el gran desafío de mejorar la salud de las personas en un escenario de continuos cambios en el perfil epidemiológico, de nuevas tecnologías en salud y de restricción de recursos. En este contexto, el sistema se ve obligado a priorizar en base a un conjunto de elementos que den cuenta del valor que tiene una nueva intervención para ese sistema de salud. La evaluación económica es uno de los elementos técnicos que informa este juicio de valor. El presente artículo introduce la evaluación económica en salud al médico clínico, desde la perspectiva de un sistema de salud que busca lograr el máximo de salud con los recursos disponibles. Se contextualiza este instrumento en el proceso de evaluación de tecnologías en salud y se discute su relevancia en el actual contexto chileno.

Correspondencia:

Dr. Manuel Espinoza, MSc PhD

Departamento de Salud Pública, Pontificia Universidad

Católica de Chile

Teléfono: 56223546807

maespinoza@med.puc.cl 


\section{Economic evaluation for decisions in Health Care: ¿what should the health professional know?}

Health care face the challenge of improving population in the context of continous epidemiological changes, development of new technologies and budget constraints. The system is forced to prioritize interventions based on a set of elements related to the value of a new intervention in that health system. Economic evaluation is one among different technical elements needed to arrive at a value judge- ment. This article to introduce the clinical physician to health economic evaluation with a perspective of obtaining the maximal health benefits given the available resources. The value of this instrument in the evaluation of health technologies and the implications for the current Chilean situation are discussed.

Keywords: Economic evaluation, Health Care. 


\section{Antecedentes:}

Los sistemas de salud no tienen la capacidad de financiar todos los servicios y tecnologías que demandan todos sus beneficiarios. Esto se explica por una parte, debido al constante cambio demográfico y epidemiológico, asi como la rápida innovación tecnológica que produce mejoras a la salud de ciertos grupos de pacientes (demanda). Por otro lado, los sistemas de salud enfrentan presupuestos limitados, incertidumbre debido a falta de información e ineficiencias de la gestión de los actuales recursos (oferta). Todo este contexto hace que los sistemas no tengan más remedio que priorizar dar coberura sólo a ciertas prestaciones.

Debido a que la priorización es un proceso que inevitablemente beneficia a algunos y posterga a otros, los sistemas de salud se ven cada vez más obligados a rendir cuentas a la población respecto de cómo se llevan a cabo estas decisiones. Esto ha motivado el desarrollo de procesos, que anclados en evidencia científica, puedan dar señales de confianza a la ciudadanía que las decisiones tomadas fueron las mejores, dados los recursos disponibles y las necesidades de la población. Este tipo de proceso se ha denominado Evaluación de Tecnologías Sanitarias (ETESA), y se ha reconocido como un campo multidisciplinario que integra información médica, económica, social, ética y legal, para informar las decisiones sobre cobertura ${ }^{1}$.

Es precisamente en este marco de ETESA que la evaluación económica en salud pasa a ocupar una posición muy relevante. En efecto, corresponde a la instancia formal, técnica y científica de abordar la pregunta si vale la pena que el sistema de salud pague por una nueva intervención en salud. Esta pregunta de valor obliga a la autoridad sanitaria a hacer explícito ciertos juicios de justicia distributiva que, en definitiva, son los fundamentos de lo que conocemos como evaluación económica.

El presente artículo tiene como propósito introducir la evaluación económica en salud al médico clínico, no simplemente como un instrumento técnico, sino como un marco de análisis que facilita la integración de información clínica y financiera con juicios de valor social. En la siguiente sección se presentan los tipos de evaluación económica, para luego profundizar en análisis de costo efectividad y su interpretación. Posteriormente, se discutirá la importancia que este tipo de evidencia tiene en el actual contexto chileno y sus desafíos futuros.

\section{Evaluación económica en salud}

\section{Tipos de Evaluación Económica}

La evaluación económica se define como un análisis comparativo de cursos alternativos de acción en términos de sus costos y consecuencias ${ }^{2}$. Se distinguen cinco tipos de evaluaciones: 1 . costo beneficio; 2 . costo efectividad; 3. costo utilidad; 4. costo minimización; 5. costo consecuencia. Cabe detacar que el análisis de impacto presupuestario no se incluye clásicamente como un tipo de evaluación económica pues corresponde a un análisis limitado a los aspectos puramente financieros. Sin embargo, será igualmente descrito en este artículo para ofrecer al lector una visión más global de la evaluación económica.

En el análisis de costo beneficio (ACB), tanto los costos como los beneficios se miden en términos monetarios. Si bien ésto no es una complicación en la estimación de costos, si lo es en la de beneficios, pues obliga a monetarizar las consecuencias o beneficios de salud. Cabe destacar que en el ACB se busca representar una medida de beneficio que sea cercana al bienestar, es decir, que incluya los beneficios en salud, pero tambien aquellos beneficios asociados que no sean estrictamente salud. La estimación de beneficios en dinero, se puede realizar utilizando métodos tales como la evaluación contingente ${ }^{3}$ o los experimentos de elección discreta ${ }^{4}$. Adicionalmente, esta valorización debe hacerse desde los individuos, pues asume que ellos son los mejores jueces de su propio bienestar, y es por lo tanto su valoración subjetiva lo que importa tomar en cuenta 5 .

El resultado del análisis se expresa como un beneficio neto por cada alternativa expresado en dinero. Esto es, simplemente, la magnitud de beneficios (en dinero) menos la magnitud en costos (también en dinero) ${ }^{6}$. La mayor ventaja de este tipo de análisis es que permite comparar estrategias de salud con prospectos en otras áreas de la economía, tales como, educación, vivienda o protección social. La mayor desventaja es que requiere valorar las consecuencias en dinero, por ejemplo, ¿cuánto vale un año de vida adicional? Esto ha generado una gran barrera para la realización de ACB en salud.

El análisis de costo efectividad (ACE) es un tipo de evaluación económica que nace como una respuesta a las críticas y complicaciones desatadas por el uso de ACB en salud; en particular en lo relativo a la monetarización de los beneficios, aunque no es la única. Dentro de otras críticas al ACB está el que no siempre el individuo es el mejor juez de su bienestar, que la estimación monetaria de beneficios depende de la capacidad de pago de las personas y que no responde una pregunta más simple y 
más directa que enfrenta el tomador de decisiones, ¿vale la pena que el sistema de salud gaste su presupuesto (y no el de otros ministerios) en una nueva intervención sanitaria? ${ }^{7}$.

Si el objetivo del sistema de salud es producir salud (y no otras dimensiones del bienestar), entonces el valor estará dado por la salud adicional que logra producir una nueva intervención comparada con la salud que se habría producido si es que los recursos necesarios para financiarla hubiesen sido destinados a otras actividades del sistema de salud ${ }^{8}$. Para hacer este juicio, el análisis requiere utilizar una métrica de salud que sea comparable entre las distintas actividades del sistema de salud, por ejemplo, años de vida o años de vida ajustados por calidad (AVAC o QALY por su sigla en inglés Quality Adjusted Life Years $)^{9}$. Así, se podrá comparar los QALYs que se esperan ganar, por ejemplo, a partir de un nuevo medicamento para tratar la insuficiencia cardíaca con los QALYs que se producen en el sistema de salud debido a la asignación de esos recursos a otras actividades del sistema como programas de salud mental u oncología.

El ACE es un tipo de evaluación donde los costos son expresados en dinero y los beneficios en unidades de salud. Sin embargo, podemos distinguir dos tipos de ACE. Primero, aquel que expresa salud como outcomes clínicos específicos a la condición en evaluación (por ejemplo, infarto agudo al miocardio) y que no es comparable con los desenlaces que producen intervenciones en otras áreas (por ejemplo, enfermedades inmunológicas). Este estudio no es informativo de asignación de recursos en el sistema de salud. Segundo, aquel que utiliza una métrica comparable entre las actividades que realiza el sistema de salud (por ejemplo, QALYs). Es precisamente este último el más utilizado en evaluación económica en el mundo, pues integra las dimensiones de cantidad de salud y calidad de vida relacionada con la salud. Si bien el lector encontrará en la literatura ambos tipos de estudios, debe tener en cuenta que solamente el segundo es el que informa decisiones para la priorización, mientras que el primero es más bien un ejercicio que se asemeja a lo que describiremos más adelante como estudio de costos y consecuencias.

El análisis de costo utilidad (ACU) es muy similar al ACE, pues comparten sus mismos fundamentos y se operacionalizan numéricamente igual. La diferencia es que en el ACU, la medida de beneficio es utilidad relacionada con la salud en vez de salud. Para efectos prácticos, la utilidad debe ser entendida como una medida de bienestar. Se distingue del ACB que este bienestar está restringido al componente que es explicado por el estado de salud alcanzado por un individuo, y no por otras dimensiones de calidad de vida. Bajo ciertos supuestos, se ha propuesto que el QALY puede ser considerado una medida de utilidad relacionada a la salud ${ }^{10}$. Sin embargo, para ello su construcción requiere que el componente de calidad de vida relacionada con la salud se haya cuantificado utilizando un instrumento de valoración llamado standard gamble ${ }^{11}$.

Debo llamar la atención al lector que la diferencia entre ACE y ACU ha sido motivo de confusión en la literatura debido a simplismos equivocados. Por ejemplo, se ha denominado ACU a todo estudio que utiliza QALYs, aún cuando en la mayoría de ellos el QALY no ha sido construido con el instrumento standard gamble. Sin embargo, esta discusión es relevante sólo en un terreno teórico, pero en la práctica no tiene mucha importancia. Cuando el interés es informar asignación de recursos en salud, lo que realmente importa es contar con alguna medida de salud, cuya única condición es que permita comparar resultados entre las distintas actividades que desempeña el sistema de salud. Para efectos de lo que sigue en este manuscrito, me referiré genéricamente como costo efectividad tanto al ACU como al ACE que informa asignación de recursos. En ambos tipos de estudios el estimador corresponde a una métrica que integra costos y beneficios, el cual será presentado formalmente en la siguiente sección.

El análisis de costo minimización (ACM) corresponde a una comparación exclusiva de los costos de las intervenciones, asumiendo que existe evidencia suficiente que demuestra que los beneficios de dichas intervenciones son iguales. Cabe destacar que cuando se comparan costos no se refiere a una comparación de precios de las intervenciones, sino que al costo esperado de manejar a un paciente con una determinada estrategia en el curso de su vida. Esto involucra a los costos directos de la tecnología pero también de los eventuales eventos adversos y complicaciones futuras. Esta perspectiva requiere modelamiento matemático que permita hacer proyecciones de los eventos que le ocurrirán a un paciente en el futuro y los costos que éstos implican. Esta aproximación a la estimación de costos es la que se utiliza para todo tipo de evaluación económica.

Se ha propuesto que el ACM debiera retirarse de la lista de los tipos de evaluación económica. El principal argumento es que nunca se tiene certeza absoluta que la efectividad de una intervención sea exactamente igual a la de 
su comparador. En la práctica la evidencia proviene de un número finito de ensayos (por ejemplo, ensayos clínicos), cada uno con incertidumbre asociada, habitualmente expresada como un intervalo de confianza. En otras palabras, toda vez que existe un error estándar mayor a cero hay incertidumbre y no se puede concluir con certeza que ambas estrategias son iguales. Por lo tanto, lo correcto es tambien considerar los beneficios esperados e incluirlos en la evaluación ${ }^{2}$.

Finalmente, el análisis de costo consecuencia (ACC) es un estudio que caracteriza por un lado costos asociados a las intervenciones en comparación, y por otro lado, un conjunto de outcomes o desenlaces en salud. La diferencia con ACE y ACU es que en el ACC no se integran costos y consecuencias en un estimador, sino que se presentan de manera separada. Este tipo de análisis es por cierto útil en la caracterización de un problema de salud, pero es limitado en ayudar al juicio de valor en el contexto de otras varias actividades que desempeña un sistema de salud.

\section{Elementos Metodológicos de la Evaluación Económica}

Si bien la evaluación económica conlleva múltiples complejidades metodológicas, hay cinco elementos básicos que deben ser siempre atendidos. Estos son: 1. la perspectiva; 2. el tiempo horizonte; 3 . la tasa de descuento; 4 . el modelamiento matemático; y 5 . la incertidumbre asociada a las decisiones.

La perspectiva del análisis define al actor que utilizará la evaluación para la toma de decisiones y su propósito. Las perspectivas más habituales son la perspectiva del sistema de salud, de un tercer pagador (por ejemplo, ISAPRES en Chile), de la sociedad y de los pacientes. Las implicancias de la perspectiva son variadas, por ejemplo, la perspectiva del sistema de salud implica que los costos considerados en el análisis sólo deben incluir costos directos que incurre el sistema. En contraste, si se adopta una perspectiva social, se deben incluir costos indirectos como, por ejemplo, los gastos de bolsillo que incurren los pacientes en traslados o copagos asi como el costo de pérdidas de productividad laboral. Aunque en principio parece que una perspectiva social es más adecuada, existen varios argumentos que sugieren que la perspectiva del sistema de salud resulta más consistente con el problema de asignación de recursos que enfrenta el sistema y con el arsenal metodológico disponible para ejecutar los análisis. Para profundizar más sobre este tema se refiere al lector a literatura especializada ${ }^{12}$.

El tiempo horizonte corresponde al tiempo sobre el cual se estimarán los costos incurridos en el manejo de un paciente y los beneficios que se desprenden se éste. Si el sistema de salud está anclado sobre la idea de producir salud en el corto pero tambien largo plazo, entonces el tiempo horizonte debiera ser el curso de vida de los pacientes. Ciertas razones que hacen acortar la definición del tiempo horizonte son: que no existan diferencias relevantes entre los grupos en comparación luego de cierto periodo; o que existan grandes dificultades para modelar el curso futuro de eventos.

La tasa de descuento es un elemento normativo de la metodología aplicada en evaluación económica. Es ampliamente reconocido que las personas prefieren tener dinero hoy sin esperar a tenerlo mañana. De manera similar, también se ha reconocido que los logros en mejorar la salud se prefieren obtener hoy en vez del futuro, en parte porque la gente que necesita salud no puede esperar. La tasa de descuento es un instrumento que representa esta preferencia temporal tanto por dinero como por resultados en salud, dando cuenta del mayor valor de ambos componentes en el presente ${ }^{13}$. Su aplicación en estudio de evaluación económica se refleja en que las estimaciones de tanto costos como beneficios se expresan en términos de su valor presente, lo cual quiere decir que una tasa de descuento ha transformado el valor del dinero a gastar en el futuro y de los resultados a obtener en el futuro, en un valor que pueda ser comparado en el presente. Para mayor información se refiere al lector al anexo sobre tasa de descuento de la guía chilena de evaluación económica ${ }^{14}$.

El modelamiento matemático corresponde a un conjunto de métodos que permiten caracterizar el curso de vida de un paciente que sufre cierta condición de salud a ser modelada ${ }^{15}$. A partir de éste se estiman eventos positivos y negativos futuros, sus costos relacionados y cómo estos afectan su calidad de vida relacionada con la salud. Existen varios tipos de modelos, sin embargo, los más utilizados son los árboles de decisión, los modelos de Markov y los modelos de microsimulación ${ }^{16}$. El modelamiento permite hacer una estimación del valor presente de los costos y beneficios esperados o promedio sobre el horizonte temporal que se ha modelado. Esta estimación depende estrictamente de los supuestos estructurales so- 
bre los cuales se ha construido el modelo, los cuales habitualmente se trabajan y validan con expertos clínicos.

Finalmente, las estimaciones de costo efectividad están asociadas a múltiples fuentes de incertidumbre ${ }^{17}$. En primer lugar la incertidumbre de primer orden corresponde a la falta de conocimiento de los factores que explican las diferencias entre (subgrupos) de individuos ${ }^{18}$, ${ }^{19}$. Por otro lado, la incertidumbre de segundo orden se debe a la imprecisión de las estimaciones de los múltiples parámetros que alimentan los modelos de decisión, tales como, probabilidades de ocurrencia de eventos en el curso de la enfermedad, probabilidades de ocurrencia de eventos adversos, efectividad, uso de recursos, costos y utilidades ${ }^{20-22}$. Su caracterización se operacionaliza en los análisis de sensibilidad determinístico y probabilístico. El primero corresponde a la exploración detallada de las variaciones del resultado de la evaluación cuando el valor de un parámetro cambia, manteniendo el resto constante (ceteris paribus). El segundo corresponde a la caracterización de los múltiples resultados de costo efectividad cuando se varian simultáneamente todos los parámetros del modelo de decisión. Este último es el más habitualmente requerido y se presenta tanto como una nube de puntos sobre el plano de costo efectividad, o como curvas de aceptabilidad de costo efectividad. Mientras el primero es simplemente una ilustración gráfica de la incertidumbre alrededor del estimador puntual, la segunda es la presentación de la probabilidad que una intervención sea costo efectiva (eje Y) en función de un umbral de costo efectividad (eje $\mathrm{X}$ ).

\section{Analisis de costo efectividad}

Tal como se mencionó anteriormente, la explicación que se presenta en esta sección aplica tanto para ACE como para ACU. Para mayor simplicidad considere la comparación entre dos alternativas: una nueva estrategia (1) y una estrategia que corresponde al cuidado estándar que ofrece el sistema de salud (0). El plano de costo efectividad que muestra la Figura 1 posiciona la estrategia estándar en el centro, y la estrategia nueva en algún cuadrante del plano. El eje X grafica la diferencia en beneficios y el eje Y grafica la diferencia en costos, ambos respecto de la estrategia estándar. El plano permite ubicar nuevas tecnologías en cuatro cuadrantes que tienen implicancias relevantes para las decisiones. La inmensa mayoria de las nuevas tecnologías que son evaluadas en salud se encuentran en el cuadrante nor este, es decir, son estrategias más beneficiosas pero tambien más caras. Sin embargo, la innovación podría proveer de alternativas que se ubiquen en otros cuadrantes.

El estimador más habitual de costo efectividad se denomina Razón de Costo Efectividad Incremental (RCEI o ICER del inglés Incremental Cost-Effectiveness Ratio), de acuerdo a la siguiente expresión:

$$
\frac{C_{1}-C_{0}}{B_{1}-B_{0}}=R C E I
$$

(1)

Donde el numerador corresponde a la diferencia de los valores presentes de los costos esperados de la nueva estrategia (C1) versus la estándar (C0), lo cual se denomina costo incremental. Gráficamente, esta magnitud se presenta en la Figura 1 como la distancia entre la estrategias nueva y estándar proyectada sobre el eje Y. El denominador corresponde a la diferencia del valor presente de los beneficios esperados (ej. QALY esperados) de la nueva estrategia (B1) versus la estándar (B0), lo cual se ilustra como la distancia entre ambas estrategias proyectadas sobre el eje X. Así la RCEI se interpreta como el costo adicional que se debe pagar para obtener una unidad adicional del beneficio, y es representada en la Figura 1 como la pendiente de la línea segmentada que une ambas estrategias. Cabe destacar que este resultado es expresado como un promedio por paciente o individuo.

Una nueva estrategia puede ser considerada como costo-efectiva si su RCEI es menor que un Umbral de Costo Efectividad $(\lambda)$, es decir:

$$
\frac{C_{1}-C_{0}}{B_{1}-B_{0}}<\lambda
$$

(2)

Este umbral de costo efectividad $(\lambda)$ es una representación del costo de oportunidad del sistema de salud. Conceptualmente corresponde al dinero que cuesta producir una unidad adicional de salud en el sistema de salud tal cual está operando. Por lo tanto, si el dinero que cuesta producir una unidad adicional de beneficio con una nueva intervención (RCEI) es menor que lo que cuesta producirla en el sistema de salud $(\lambda)$, es coherente adoptar la nueva intervención. Eso significa que una nueva intervención sea costo-efectiva. 


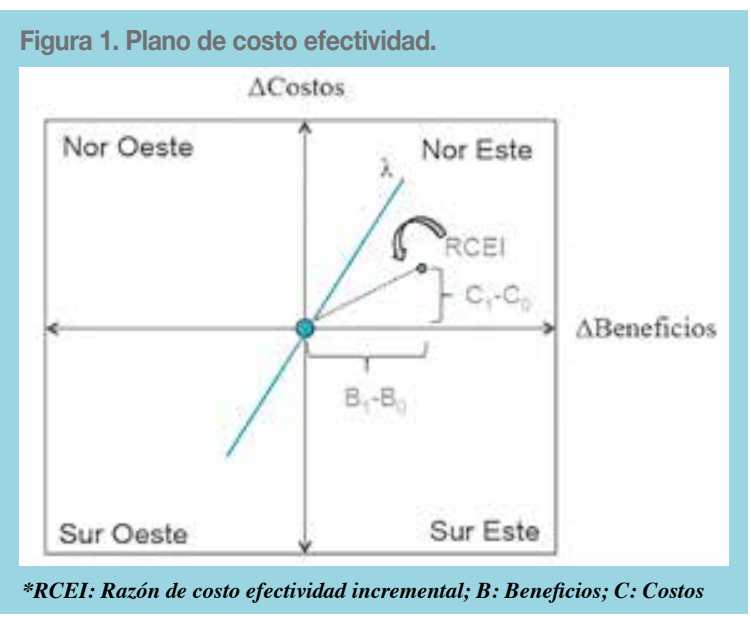

La Figura 1 representa el umbral de costo efectividad como una linea recta que pasa por el centro del plano, cuya pendiente corresponde exactamente a su magnitud. Por lo tanto, todas aquellas estrategias ubicadas por debajo de esta línea, corresponderán a alternativas costo-efectivas, mientras que todas aquellas por arriba del umbral no son costo-efectivas. Esta representación ilustrativa es coherente con la regla de decisión presentada en la ecuación 2.

Es importante destacar que el costo-efectividad no es una comparación de costos y salud, sino que una comparación entre salud y salud. Esto es, la salud adicional ganada por la nueva estrategia versus la salud que se ha dejado de producir en el sistema de salud como consecuencia de los costos adicionales (costo de oportunidad). Este concepto se demuestra con un simple rearreglo de la ecuación (2):

$$
\frac{C_{1}-C_{0}}{\lambda}<B_{1}-B_{0}
$$

(3)

Donde ambas expresiones de la desigualdad están en términos de beneficios en salud. La Tabla 1 muestra una aplicación de este concepto. La comparación de más de 2 estrategias se lleva a cabo mediante el denominado análisis incremental, el cual es una extensión de la explicación presentada en este artículo. Se refiere al lector a consultar la guía chilena de evaluaciones económicas para profundizar en este análisis ${ }^{23}$.

\section{Importancia de la evaluación económica en chile y sus desafios futuros}

La evaluación económica en salud, en particular el análi- sis de costo efectividad (ACE/ACU), ha sido reconocido como un elemento relevante para informar la toma de decisiones sobre cobertura en salud. El primer reconocimiento en el marco normativo chileno está en la Ley que creó el Régimen de Garantías Explícitas en Salud, donde se expresa que “...considerará el desarrollo de estudios con el objetivo de determinar un listado de prioridades en salud y de intervenciones que consideren la situación de salud de la población, la efectividad de las intervenciones, su contribución a la extensión o a la calidad de vida y, cuando sea posible, su relación costo efectividad" 24 . Posteriormente, el Ministerio de Salud elaboró una Guía Metodológica para la Elaboración de Evaluaciones Económicas en Salud ${ }^{23}$. Más recientemente, la evaluación económica se reconoce como un elemento que debe estar presente en la evaluación de tecnologías que están siendo consideradas para entrar al Sistema de Protección Financiera para Diagnósticos y Tratamientos de Alto Costo, también denominada Ley Ricarte Soto ${ }^{25}$.

Debido a la condicionalidad que expresa la Ley del Régimen GES en términos de usar costo-efectividad en la medida que exista esta evidencia, nunca se generó una real necesidad por conducir dichos estudios ni tampoco por formar profesionales capacitados. Más bien se vió postergado en comparación con el crecimiento de competen-

Tabla 1.- Interpretación del análisis de costo efectividad (estudio hipotético)

Un estudio de costo-efectividad comparó un nuevo medicamento (A) para el tratamiento de la insuficiencia cardíaca con baja fracción de eyección comparado con la terapia habitual (B). El estudio adoptó una perspectiva del sistema público de salud y estimó el valor presente de los costos incurridos por el sistema y desenlaces ocurridos al paciente durante su curso de vida, utilizando una tasa de descuento indiferenciada del $3 \%$. Se utilizó un modelo de Markov que permitió estimar los efectos al largo plazo. La efectividad se obtuvo de un ensayo clínico multicéntrico que incluyó ambos comparadores. Los resultados mostraron:

\begin{tabular}{|l|l|l|l|}
\hline & Costos & QALYS & RCEI \\
\hline Medicamento A & $\$ 8.754 .867$ & 5.5 & \\
\hline Medicamento B & $\$ 5.567 .245$ & 5.3 & \\
\hline Incremental & $\$ 3.187 .622$ & 0.2 & $\$ 15.938 .110$ \\
\hline $\begin{array}{l}\text { La interpretación de la RCEI es que por cada QALY adicional el sistema de- } \\
\text { berá pagar \$15.938.110, lo cual comparado con un umbral de referencia de } \\
\text { \$10.500.000 (I PIB per cápita) permite concluir que el nuevo medicamento } \\
\text { no es costo efectivo. }\end{array}$ \\
\hline
\end{tabular}


cias en medicina basada en evidencia (MBE). Por cierto, la urgencia de contar con decisiones basadas en evidencia clínica le hizo sentido tanto a la autoridad sanitaria como al mundo médico, que se vió rápidamente incentivado a recibir formación para participar de estos procesos de decisión y de la construcción de guías de práctica clínica. En tanto, la formación en evaluación económica respondió al interés personal de contados profesionales que vieron en esta área un potencial de desarrollo profesional, en buena parte motivados por la experiencia internacional. Es importante destacar que en Chile la inclusión de una nueva intervención en una guía de práctica clínica no implica su cobertura. Distinto a lo que ocurre en otras juridicciones del mundo, en Chile la Guía Clínica es un reflejo del lex artis que debiese ser aplicado en el país, pero que no incluye en su elaboración elementos de eficiencia en la asignación de recursos en el sistema de salud. Por lo tanto, en la práctica mucho de lo que está recomendado en las guías clínicas chilenas no está en las canastas de cobertura.

De acuerdo a lo observado en el quehacer chileno, las decisiones de cobertura se toman de manera desagregada. En la inmensa mayoría de las decisiones de cobertura participa el asegurador público, Fondo Nacional de Salud (FONASA), quien ciertamente tiene una contraparte en el Ministerio de Hacienda cuando se trata de recursos adicionales. Por otro lado, las ISAPRES toman sus propias decisiones a discreción sin ninguna guía técnica específica. Sin embargo, habitualmente se considera como requerimiento que la tecnologia esté en las guías clínicas chilenas. Finalmente el sistema de las fuerzas armadas también tiene autonomía para sus decisiones, las cuales son a discreción.

Por cierto que el Ministerio de Salud tiene un rol importante en este ejercicio decisor, especialmente en lo relativo a las coberturas del GES, y ahora más recientemente en las que ingresan a la Ley Ricarte Soto. Por otro lado, tiene implicancias indirectas en el sector privado mediante la generación de guías clínicas. Lamentablemente los procesos de evaluación y toma de decisión no están bien estructurados, no tienen tiempos establecidos claros, la forma de integrar los criterios de priorización no está descrita y los ejercicios de priorización no son lo suficientemente transparentes para conducir un adecuado escrutinio público. Es de esperar que el proceso de evaluación y decisión que propone la Ley Ricarte Soto y que aún espera ser implementado, sea un ejercicio tomado seriamente en cuenta por la autoridad sanitaria.

El camino que debe seguir Chile es avanzar hacia la institucionalización de un proceso de evaluación estructurado, científicamente riguroso y transparente en que la ciudadanía pueda confiar. Esto implica la creación de una agencia de Evaluación de Tecnologías en Salud, autónoma e independiente de influencias políticas o de otra índole, que puedan desviar las decisiones del objetivo que es lograr la máxima mejoría de salud de la población con los recursos disponibles ${ }^{26}$. Para avanzar en esta línea se han generado propuestas tanto desde el Ministerio de Salud como desde las Universidades que aún no se han materializado como política pública ${ }^{27}$.

Finalmente, desde lo más terrenal, es urgente mantener el creciente desarrollo de evaluaciones económicas que se ha venido generando en el país los últimos años y que se ha visto impulsada con la promulgación de la Ley Ricarte Soto. Esto requiere una colaboración más estrecha entre los profesionales médicos y los economistas de la salud que desarrollan este tipo de estudios. Por cierto que el entrenamiento de profesionales clínicos en temas técnicos de evaluación económica, como lo ha sido en MBE por ejemplo, sería un importante catalizador tanto de la generación de estudios como del impacto en los procesos de decisión sobre cobertura en Chile. 


\section{Referencias}

1. BANTA D. What is technology assessment? International journal of technology assessment in health care. 2009;25:7-9.

2. DRUMMOND M, SCULPHER M, CLAXTON K, STODDART G, TORRANCE G. Methods for the Economic Evaluation of Health Care Programmes. 4th ed: Oxford Univeristy Press; 2015.

3. KLOSE T. The contingent valuation method in health care. Health Policy. 1999;47:97-123.

4. RYAN M, GERARD K. Using discrete choice experiments to value health care programmes: current practice and future research reflections. Appl Health Econ Health Policy. 2003;2:55-64.

5. BROUWER WB, CULYER AJ, VAN EXEL NJ, RUTTEN FF, Welfarism vs. extra-welfarism. J Health Econ. 2008;27:325-38.

6. SUGDEN R, WILLIAMS A. The principles of practical cost-benefit analysis. Oxford: Oxford University Press; 1978.

7. BROUWER WB, KOOPMANSCHAP MA. On the economic foundations of CEA. Ladies and gentlemen, take your positions! J Health Econ. 2000;19:439-59.

8. PALMER S, RAFTERY J. Economic Notes: opportunity cost. Bmj. 1999;318:1551-2.

9. BRAZIER J, RATCLIFFE J, SALOMON J, TSUCHIYA A. Measuring and valuing health benefits for economic evaluation. Oxford: Oxford University Press; 2007.

10. PLISKIN J, SHEPARD D, WEINSTEIN MC. Utility functions for life years and health status. Operations Research. 1980;28:206-24.

11. DOLAN P, GUDEX C, KIND P, WILLIAMS A. Valuing health states: a comparison of methods. J Health Econ. 1996;15:209-31.

12. CLAXTON K, WALKER S, PALMER S, SCULPHER M. Appropriate perspectives for Healthcare Decisions. CHE Research Paper. 2010;54.
13. TORGERSON DJ, RAFTERY J. Economic notes. Discounting. Bmj. 1999;319:914-5.

14. CASTILlO-RIQUELME M, ESPINOZA MA. El uso y la determinación de la tasa de descuento en Evalauciones Económicas de intervenciones en Salud. . Santiago: Ministerio de Salud, 2011.

15. BRIGGS A, SCULPHER MJ, CLAXTON K, editors. Decision Modelling for Health Economic Evaluation. Gosport, Hampshire: Oxford University Press; 2006.

16. PHILIPS Z, GINNELLY L, SCULPHER M, CLAXTON K, GOLDER S, RIEMSMA R, et al. Review of guidelines for good practice in decision-analytic modelling in health technology assessment. Health Technology Assessment (Winchester, England). 2004;8: iii-iv, ix-xi, 1-158.

17. BRIGGS AH, WEINSTEIN MC, FENWICK EA, KARNON J, SCULPHER MJ, PALTIEL AD. Model parameter estimation and uncertainty: a report of the ISPOR-SMDM Modeling Good Research Practices Task Force--6. Value in health : the journal of the International Society for Pharmacoeconomics and Outcomes Research. 2012;15:835-42.

18. ESPINOZA MA, MANCA A, CLAXTON K, SCULPHER MJ. The value of heterogeneity for cost-effectiveness subgroup analysis: conceptual framework and application. Med Decis Making. 2014;34:951-64.

19. ESPINOZA M, SCULPHER M, MANCA A, BASU A. Heterogeneity in Decision-Making about Health Interventions. In: Culyer A, editor. Encyclopedia of Health Economics: Elsevier; 2014.

20. O'HAGAN A, MCCABE C, AKEHURST R, BRENNAN A, BRIGGS A, CLAXTON K, et al. Incorporation of uncertainty in health economic modelling studies. Pharmacoeconomics. 2005;23:529-36.

21. GLICK HA, BRIGGS AH, POLSKY D. Quantifying stochastic uncertainty and presenting results of cost-effectiveness analyses. Expert Rev Pharmacoecon Outcomes Res. 2001;1:25-36. 
22. BRIGGS AH. Statistical approaches to handling uncertainty in health economic evaluation. Eur J Gastroenterol Hepatol. 2004;16:551-61.

23. MINISTERIO DE SALUD DE CHILE. Guia metodológica para la evaluación de intervenciones en salud en Chile. In: Departamento de Economía de la Salud, editor. Santiago 2013.

24. Establece un Regimen de Garantías en Salud, 2004.

25. Crea un Sistema de Protección Financiera para Diagnósticos y Tratamientos de Alto Costo y Rinde Homenaje Póstumo a
Don Luis Ricarte Soto Gallegos, 2015.

26. ESPINOZA MA. La urgente necesidad de un sistema de evaluación de intervenciones en salud Rev Med Chil. 2016;144: 903-905.

27. Comisión Nacional de Evaluación de Tecnologías Sanitarias. Propuesta de un modelo de implementación e institucionalización de la Evaluación de Tecnologías Sanitarias en Chile. Disponible en: http://web.minsal.cl/sites/default/files/files/ InformeFinalPropuestaETESAChile.pdf. Santiago2014. 